\title{
The Protective Effect of Short-term Infusion Regimens with Sodium Bicarbonate and Theophylline against Contrast Induced Nephropathy in Rats
}

\author{
Gihan B. Azab and Rabab Nabil Hafiz1
}

${ }^{1}$ Departments of Forensic Medicine \&Clinical Toxicology, Faculty of Medicine, Ain Shams University, Cairo, Egypt.

\begin{abstract}
Contrast-induced nephropathy (CIN) is a common condition associated with serious adverse outcomes. It may be preventable because its risk factors are well characterized and the timing of renal insult is commonly known in advance. The optimal strategy to prevent contrast nephropathy has not been established. The AIM of this study is to compare the efficacy of short-term infusion regimens of sodium bicarbonate, theophylline and the combination of both for prevention of contrast-induced nephropathy in rats. METHODS: This work was conducted on seventy adult male albino rats. They were classified into seven groups. Group I: control group; Group II: received $1 \mathrm{~mL}$ intravenous bicarbonate (8.4\%); Group III: received $15 \mathrm{~mL} / \mathrm{kg}$ intra-peritoneal theophylline; Group IV: received $6 \mathrm{~mL} / \mathrm{kg}$ intravenous urografin; Group V: intravenous bicarbonate three hours before urografin; Group VI: intra-peritoneal theophylline one hour before urografin; Group VII: bicarbonate three hours and theophylline one hour before urografin injection. Baseline blood samples were collected and analyzed for biochemical parameters then after waiting 48 hours for the development of contrast nephropathy the rats were sacrificed and biochemical parameters were re-examined: serum creatinine, blood urea nitrogen (BUN), creatinine clearance and fractional excretion of sodium $\left(\mathrm{FENa}^{+}\right)$. Light microscopic examination for the kidneys sections was done. RESULTS: after 48 hours water deprivation, highly significant decrease in mean body weight was found in all groups when compared with the baseline mean values. On comparing baseline with the after experiment parameters mean values within each group, there was significant increase in the mean values of serum creatinine and BUN and significant decrease in the creatinine clearance and fractional excretion of sodium percentage in groups group IV, group V and group VI; while, group VII showed insignificant difference between baseline and after experiment in all the studied parameters. Comparing after experiment parameters between group IV and control group showed a highly significant increase in the mean values of serum creatinine, BUN and significant decrease in the creatinine clearance and fractional excretion of sodium percentage. Furthermore, group V and group VI showed marked amelioration of all after experimental parameters but it still shows significant difference when compared with the control group. Group VII showed insignificant difference in all parameters when compared with the control group. Histopathological examination of the examined specimens from group IV showed severe damage consisting of tubular necrosis and protein cast, tubule dilatation, intra-tubular obstruction by protein casts. The examined kidney specimens from group V and group VI showed mild damage while the percentage of pathological changes were significantly decreased when compared with group IV. Sections from group VII showed marked improvement of all changes. CONCLUSION: urografin injection resulted in marked nephrotoxicity manifested biochemically and histopathologically. Pre-treatment with sodium bicarbonate or theophylline partially ameliorated the CIN. While, combined administration of sodium bicarbonate and theophylline before urografin injection showed marked improvement of renal function and histopathological examination in rats.
\end{abstract}

\section{Introduction}

$\mathrm{I}$ ntravenous administration of contrast dye can lead to a contrast-induced nephropathy (CIN) that begins soon after its injection (Abouzeid and Mosbah, 2016; Lefel et al.,2016).CIN remains the third most common cause of hospital-acquired acute renal failure (Yeganehkhah et al., 2014). It is associated with prolonged hospitalization, need for renal replacement therapy and increase in occurrence 
of short- and long-term mortality (Rudnick and Feldman, 2008; Mahmoodi et al.,2014). It is highly prevalent especially, in patients with well-known risk factors including old age, dehydration, diabetic nephropathy and congestive heart failure (Boucek et al., 2013).

As the diagnostic procedures using contrast dye has been increasing; the patient population is aging; diabetes and chronic kidney disease are becoming more common, so CIN is likely to remain a significant challenge in the future (Hörl, 2009).

The pathophysiological mechanisms of the development of CIN remain unclear. The proposed mechanisms are outer-medullary hypoxia arising from an imbalance of local vasoconstrictive and vasodilatory influences which seems to be mediated partly by adenosine. High osmolarity contrast dye may also have a direct cytotoxic effect that operates through the generation of reactive oxygen species within the acid environment of the renal medulla (Barlak etal., 2010; Abouzeid and Mosbah ,2016).

No current treatment can reverse CIN once it occurs, the best treatment is prevention. Clinically many types of prophylaxis have been used to prevent CIN, including patient selection, minimizing the amount of contrast agent, usage of non ionic lowosmolar contrast media and extracellular volume expansion with isotonic saline before and after exposure to contrast agents (Kwok et al.,2013; Weisbord et al., 2013; Au et al.,2014).

In low risk patients intravenous volume expansion with isotonic saline twelve hours before and after contrast medium infusion has demonstrated effectiveness in the prevention of CIN (Hörl, 2009). However, sufficient hydration may be impossible in patients with a concomitant decrease in renal blood flow (e.g. congestive heart failure) (Schiffl, 2015). Moreover, there is no evidence regarding the several short-term prophylaxis protocols against CIN that may be most feasibly convenient in emergency and outpatients settings (Kama et al., 2014). So in high risk patients additional prophylactic measures are needed.

Alkalinization with sodium bicarbonate have been proposed as a mean of reducing free-radical mediated renal injury, but its effectiveness in patients remains uncertain (Boucek et al.,2013; Mahmoodi et al.,2014;Schiffl ,2015; Lefel et al.,2016).

In the past few years, experiments have shown that renal adenosine acts as a vasoconstrictive metabolite in the kidney contributing to the fall in glomerular filtration rate. Vasoconstriction produced by adenosine can be inhibited by the nonspecific adenosine receptor antagonist, theophylline. Several trials with theophylline have shown some evidence to suggest that it may reduce incidence of CIN. Although there is, limitations in the studies quality and heterogeneity preclude any firm recommendations (Kwok et al.,2013; Arabmomeni et al., 2015).

The aim of this study is to compare the efficacy of short-term infusion regimens of sodium bicarbonate, theophylline and the combination of both for prevention of contrast-induced nephropathy in rats.

\section{Materials and methods}

This work was conducted on seventy male adult albino rats weighing (140-160 grams each). They were kept in special animal cages under standardized conditions with free water supply and balanced diet.

Ethical consideration of the study: experimental procedure was performed in accordance with the guide of the care and use of laboratory animal's protocol approved by the Ethical Committee of Ain Shams University. The ethically approved conditions used by animal housing and handling were considered. The experimental protocol used followed the regulation for administration and painless sacrifaction for experimental animals.

\section{Materials}

- Ionic high-osmolar contrast medium: meglumine/sodium diatrizoate (Urografin $76 \%)$, iodine concentration $(370 \mathrm{mg} / \mathrm{mL})$ (Bayer). $1 \mathrm{~mL}$ Urografin $76 \%$ contains $0.1 \mathrm{~g}$ sodium amidotrizoate and $0.66 \mathrm{~g}$ meglumine amidotrizoate in aqueous solution. Given at a dose of $6 \mathrm{ml} / \mathrm{kg}$ into the tail vein over a period of 2 minutes. The dose of contrast medium is a standard for clinical use and for other relevant experiments in rat models (Toprak et al., 2008).

- Sodium bicarbonate was purchased from Otsuka Co. It was injected into the tail vein at a dose of $1 \mathrm{~mL}$ (8.4\%) (Barlak et al., 2010).

- Theophylline was purchased from Memphis Co. It was injected intra-peritoneal at a dose of $15 \mathrm{mg} / \mathrm{kg}$ (Ozturk et al., 2015).

\section{Animal grouping}

The rats were randomized into seven groups; ten rats each.

Group I: Control group.

Group II: received intravenous bicarbonate.

Group III: received intra-peritoneal theophylline.

Group IV: received intravenous urografin.

Group V: received bicarbonate three hours before urografin.

Group VI: received theophylline one hour before urografin.

Group VII: received bicarbonate three hours and theophylline one hour before urografin.

\section{Study protocol}

Rats were kept in individual cages to collect 24 hours urine. They were weighed once a day. Baseline blood samples were collected from the tail vein under ether anaesthesia and analyzed for biochemical parameters. All rats were deprived from water for 48 hours (to potentiates the vasoconstrictive effects of contrast dye (Toprak et al.,2008; Barlak et al.,2010).The contrast medium (urografin) was injected to groups IV, V, VI and VII. In group II, intravenous sodium bicarbonate was administered. In groups $\mathrm{V}$ and VII sodium bicarbonate injected three hours before IV urografin. In group III theophylline, was injected intra-peritoneal. In groups VI and VII theophylline injected one hour before urografin administration. After waiting 48 hours for the development of contrast nephropathy (Mahmoodi et al., 2014), rats were housed in the cages 
again for $24 \mathrm{~h}$ urine collection. Then the rats were sacrificed and biochemical parameters were reexamined. The final blood sample was withdrawn from the abdominal aorta at the end of the study.

\section{Biochemical parameters}

- Serum creatinine and blood urea nitrogen (BUN) were measured by colorimetric method according to Lawrence and Robert (1993). The assay kits were purchased from Alkane Company.

- Creatinine clearance was calculated by $U \times$ $V / P$ [where $U=$ urine creatinine $(\mathrm{mg} / \mathrm{dl}), V=$ urine volume $(\mathrm{ml} / \mathrm{min} / 100 \mathrm{~g})$, and $P=$ serum creatinine $(\mathrm{mg} / \mathrm{dl})]$, and was expressed as $\mathrm{ml} / \mathrm{min} / 100 \mathrm{~g}$ body weight (Hafeez et al.,2016).

- Fractional excretion of sodium $\left(\mathrm{FENa}^{+}\right)$was calculated as: $\mathrm{FENa}^{+}(\%)=$ (urine sodium/plasma sodium) $\times$ (plasma creatinine/urine creatinine) $\times 100$ (Legrand et al., 2016).

\section{Histopathological examination}

Both kidneys were excised immediately and were placed in $10 \%$ formaldehyde for histopathological examination. Histological slides were prepared and then counterstained with haematoxylin and eosin (H\&E) (Toprak et al., 2008).

\section{Statistical analysis}

The statistical analysis was performed using a standard SPSS (Statistical Package for Social Science) software package, version 20 (Chicago, IL). Data were expressed as (mean $\pm \mathrm{SD}$ ). Student " $\mathrm{t}$ " test, ANOVA, One Way Statistical Analysis and Chi square test $\left(\mathrm{X}^{2}\right)$ were used to analyze the data, with $p<0.05$ considered statistically significant (Taylor, 1990).

\section{Results}

All rats survived until the end of the experiment. ANOVA One way Analysis comparing the baseline mean body weights between all groups, showed no significant difference, in addition comparing the mean body weights after water deprivation between all groups showed no significant difference. Student ' $t$ ' test showed a highly significant decrease in mean body weight after water deprivation when compared with the baseline mean values in all groups (table1).

\section{Biochemical Results}

As regards the mean values of baseline serum creatinine, BUN, creatinine clearance and fractional excretion of sodium, there were insignificant differences between all the groups when compared with the control group (table 2).

On comparing baseline with the after experiment parameters mean values within each group (Table 3). There was insignificant difference as regards all tested parameters in groups I (control), II (rats received bicarbonate) and group III (rats received theophylline).

Rats received urografin (group IV), bicarbonate administration before urografin (group V) and theophylline administration before urografin (group VI) showed significant increase in the mean values of serum creatinine and BUN and significant decrease in the creatinine clearance and fractional excretion of sodium percentage. Group VII showed insignificant difference between the baseline and after experiment parameters.

By applying ANOVA One Way statistical analysis, comparing the mean values of the biochemical parameters after the experiment between all groups and control group (table 4). Group IV (urografin) showed highly significant increase in the mean values of serum creatinine and BUN and significant decrease in the creatinine clearance and fractional excretion of sodium percentage when compared with the control group.

Bicarbonate administration before urografin (group V) and theophylline administration before urografine (group VI) markedly ameliorated the elevated serum creatinine, BUN, the decrease in the creatinine clearance and fractional excretion of sodium percentage but it still shows significant difference when compared with the control group.

Administration of bicarbonate three hours and theophylline one hour before urografin injection (group VII) showed insignificant difference in all parameters when compared with the control group.

\section{Histopathological Results}

Examination of sections of rat kidneys by light microscope showed that administration of bicarbonate (group II) and theophylline (Groups III) did not show any difference from group I (control group). They showed classical histological structure of the renal cortex and medulla. The cortex was formed of nephrons that consisted of renal corpuscles proximal and distal convoluted tubules. Peri-tubular capillaries were seen between the renal tubules. Renal corpuscle was formed of glomerular capillaries and Bowman's corpuscle. Proximal convoluted tubules were lined by acidophilic cuboidal cells and showed apical brush border. Distal convoluted tubules were also lined by acidophilic cuboidal cells. The medulla of the kidney is formed of collecting tubules and loop of Henle (figure 1).

The examined specimens from rats received urografin (group IV) revealed severe damage consisting of tubular necrosis and protein cast. Damage was observed in the proximal tubular epithelial cells in the outer stripe of the outer medulla and cortex. The marked structural damage, included tubule dilatation, necrosis, and intra-tubular obstruction by protein casts, was present. Moreover, the percentage of tubular necrosis, protein casts, medullary congestion and interstitial edema were markedly different from those in the control group (Table 5 and Figure 2).

The examined kidney specimens from rats received pre-treatment with sodium bicarbonate (group V) and pre- treatment with theophylline (group VI) showed mild damage consisting of tubular necrosis, protein cast, medullary congestion and interstitial edema. The percentage of pathological changes was significantly decreased when compared with group IV (Table 5 and figures $3 \& 4$ ). 
Kidney sections obtained from rats received pre-treatment with both bicarbonate and theophylline before urografin (group VII) showed marked improvement of all changes as regards tubular necrosis, protein cast, medullary congestion and interstitial edema (Table 5 and figure 5).

Table 1: ANOVA, one way Statistical Analysis and Student ' $t$ ' test comparing body weight (BW) among group I: (control group); group II: (received intravenous bicarbonate); group III: (received intra-peritoneal theophylline); group IV: (received intravenous urografin); group V: (intravenous bicarbonate three hours before urografin); group VI: (intra-peritoneal theophylline one hour before urografin); group VII: (bicarbonate three hours and theophylline one hour before urografin injection). (10 rats/group).

\begin{tabular}{|c|c|c|c|c|c|c|c|c|}
\hline & Group I & $\begin{array}{c}\text { Group } \\
\text { II }\end{array}$ & $\begin{array}{c}\text { Group } \\
\text { III }\end{array}$ & $\begin{array}{c}\text { Group } \\
\text { IV }\end{array}$ & $\begin{array}{c}\text { Group } \\
\text { V }\end{array}$ & $\begin{array}{c}\text { Group } \\
\text { VI }\end{array}$ & $\begin{array}{c}\text { Group } \\
\text { VII }\end{array}$ & ANOVA \\
\hline $\begin{array}{l}\text { Baseline } \\
\text { BW (gm)(M } \pm \text { SD) }\end{array}$ & $\begin{array}{l}147.8 \pm \bullet \\
12.5\end{array}$ & $\begin{array}{l}149.1 \pm \\
11.9\end{array}$ & $\begin{array}{l}144.9 \pm \\
13.1\end{array}$ & $\begin{array}{l}146.8 \pm \\
12.2\end{array}$ & $\begin{array}{l}145.1 \pm \\
15.1\end{array}$ & $\begin{array}{l}148.5 \pm \\
11.7\end{array}$ & $\begin{array}{l}149.5 \pm \\
11.2\end{array}$ & $>0.05$ \\
\hline $\begin{array}{l}\text { BW after water } \\
\text { deprivation }(M \pm S D)\end{array}$ & $\begin{array}{l}117.1 \pm \\
13.2\end{array}$ & $\begin{array}{l}119.2 \pm \\
11.7 \\
\end{array}$ & $\begin{array}{l}124.7 \pm \\
12.4 \\
\end{array}$ & $\begin{array}{l}120.5 \pm \\
12.6\end{array}$ & $\begin{array}{l}116.4 \pm \\
13.1 \\
\end{array}$ & $\begin{array}{l}118.4 \pm \\
11.7 \\
\end{array}$ & $\begin{array}{l}119.9 \pm \\
11.5 \\
\end{array}$ & $>0.05$ \\
\hline t & 5.3 & 5.6 & 3.5 & 4.7 & 4.5 & 5.7 & 5.8 & \\
\hline $\mathbf{P}$ & $<0.01$ & $<0.01$ & $<0.05$ & $<0.01$ & $<0.01$ & $<0.01$ & $<0.01$ & \\
\hline
\end{tabular}

(M $\pm S D):$ mean \pm standard deviation; $t$ : student ' $t$ ' test; $p<0.05$ : significant difference; $P<0.01$ : highly significant difference.

Table 2: ANOVA, One Way Statistical Analysis of baseline serum creatinine, blood urea nitrogen (BUN), creatinine clearance and fractional excretion of sodium (FENa ${ }^{+}$) in group I: (control group); group II: (received intravenous bicarbonate); group III: (received intra-peritoneal theophylline); group IV: (received intravenous urografin); group V: (intravenous bicarbonate three hours before urografin); group VI: (intra-peritoneal theophylline one hour before urografin); group VII: (bicarbonate three hours and theophylline one hour before urografin injection). (10 rats/group).

\begin{tabular}{|c|c|c|c|c|c|c|c|c|}
\hline parameters & Group I & $\begin{array}{l}\text { Group } \\
\text { II }\end{array}$ & $\begin{array}{l}\text { Group } \\
\text { III }\end{array}$ & $\begin{array}{l}\text { Group } \\
\text { IV }\end{array}$ & $\begin{array}{c}\text { Group } \\
\text { V }\end{array}$ & $\begin{array}{c}\text { Group } \\
\text { VI }\end{array}$ & $\begin{array}{l}\text { Group } \\
\text { VII }\end{array}$ & $\mathbf{P}$ \\
\hline $\begin{array}{l}\text { Serum Creatinine } \\
\text { (mg/dl) }\end{array}$ & $\begin{array}{l}0.45 \pm \\
0.03\end{array}$ & $\begin{array}{l}0.45 \pm \\
0.05\end{array}$ & $\begin{array}{l}0.46 \pm \\
0.04\end{array}$ & $\begin{array}{l}0.43 \pm \\
0.05\end{array}$ & $\begin{array}{l}0.45 \pm \\
0.02\end{array}$ & $\begin{array}{l}0.45 \pm \\
0.04\end{array}$ & $\begin{array}{l}0.46 \pm \\
0.05\end{array}$ & $>0.05$ \\
\hline $\begin{array}{l}\text { BUN } \\
(\mathrm{mg} / \mathrm{dl})\end{array}$ & $\begin{array}{l}24.2 \pm \\
2.13\end{array}$ & $\begin{array}{l}25.0 \pm \\
1.94\end{array}$ & $\begin{array}{l}25.3 \pm \\
1.94\end{array}$ & $\begin{array}{l}24.5 \pm \\
2.60\end{array}$ & $\begin{array}{l}25.1 \pm \\
2.33\end{array}$ & $\begin{array}{l}24.9 \pm \\
2.94\end{array}$ & $\begin{array}{l}25.7 \pm \\
1.05\end{array}$ & $>0.05$ \\
\hline $\begin{array}{l}\text { creatinine clearance } \\
(\mathrm{ml} / \mathrm{min} / \mathbf{1 0 0 g})\end{array}$ & $\begin{array}{l}0.96 \pm \\
0.21\end{array}$ & $\begin{array}{l}0.99 \pm \\
0.18\end{array}$ & $\begin{array}{l}1.03 \pm \\
0.19\end{array}$ & $\begin{array}{l}0.97 \pm \\
0.17\end{array}$ & $\begin{array}{l}0.99 \pm \\
0.23\end{array}$ & $\begin{array}{l}0.98 \pm \\
0.13\end{array}$ & $\begin{array}{l}1.06 \pm \\
0.18\end{array}$ & $>0.05$ \\
\hline $\mathbf{F E N a}^{+}$ & $\begin{array}{l}0.98 \pm \\
0.23\end{array}$ & $\begin{array}{l}1.03 \pm \\
0.19\end{array}$ & $\begin{array}{l}0.99 \pm \\
0.25\end{array}$ & $\begin{array}{l}0.97 \pm \\
0.07\end{array}$ & $\begin{array}{l}0.99 \pm \\
0.11\end{array}$ & $\begin{array}{l}1.04 \pm \\
0.10\end{array}$ & $\begin{array}{l}1.01 \pm \\
0.12\end{array}$ & $>0.05$ \\
\hline
\end{tabular}

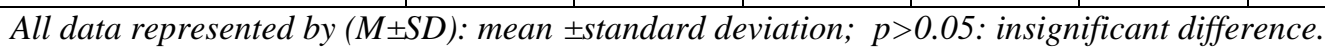


Table 3: Student ' $t$ ' test comparing baseline and after experiment serum creatinine, blood urea nitrogen (BUN), creatinine clearance and fractional excretion of sodium (FENa ${ }^{+}$) within group I: (control group); group II: (received intravenous bicarbonate); group III: (received intra-peritoneal theophylline); group IV: (received intravenous urografin); group V: (intravenous bicarbonate three hours before urografin); group VI: (intraperitoneal theophylline one hour before urografin); group VII: (bicarbonate three hours and theophylline one hour before urografin injection). (10 rats/group).

\begin{tabular}{|c|c|c|c|c|c|c|c|}
\hline parameters & Group I & $\begin{array}{c}\text { Group } \\
\text { II }\end{array}$ & $\begin{array}{c}\text { Group } \\
\text { III }\end{array}$ & $\begin{array}{c}\text { Group } \\
\text { IV }\end{array}$ & Group V & Group VI & $\begin{array}{c}\text { Group } \\
\text { VII }\end{array}$ \\
\hline $\begin{array}{l}\text { Baseline } \\
\text { Serum Creatinine (mg/dl) }\end{array}$ & $\begin{array}{l}0.45 \pm \\
0.03\end{array}$ & $\begin{array}{l}0.45 \pm \\
0.05\end{array}$ & $\begin{array}{l}0.46 \pm \\
0.04\end{array}$ & $\begin{array}{l}0.43 \pm \\
0.05\end{array}$ & $\begin{array}{l}0.45 \pm \\
0.02\end{array}$ & $\begin{array}{l}0.45 \pm \\
0.04\end{array}$ & $\begin{array}{l}0.46 \pm \\
0.05\end{array}$ \\
\hline $\begin{array}{l}\text { Serum Creatinine } \\
\text { (mg/dl) } \\
\text { after experiment }\end{array}$ & $\begin{array}{l}0.46 \pm \\
0.05\end{array}$ & $\begin{array}{l}0.46 \pm \\
0.05\end{array}$ & $\begin{array}{l}0.44 \pm \\
0.03\end{array}$ & $\begin{array}{l}2.53 \pm \\
0.15\end{array}$ & $\begin{array}{l}0.79 \pm \\
0.25\end{array}$ & $\begin{array}{l}0.67 \pm \\
0.23\end{array}$ & $\begin{array}{l}0.47 \pm \\
0.04\end{array}$ \\
\hline$t$ & 0.5 & 0.4 & 1.2 & 42 & 4.2 & 2.9 & 0.49 \\
\hline $\mathbf{p}$ & $>0.05$ & $>0.05$ & $>0.05$ & $<0.01$ & $<0.05$ & $<0.05$ & $>0.05$ \\
\hline $\begin{array}{l}\text { Baseline } \\
\text { BUN } \\
(\mathbf{m g} / \mathbf{d l})\end{array}$ & $\begin{array}{l}24.2 \pm \\
2.13\end{array}$ & $\begin{array}{l}25.0 \pm \\
1.94\end{array}$ & $\begin{array}{l}25.3 \pm \\
1.94\end{array}$ & $\begin{array}{l}24.5 \pm \\
2.60\end{array}$ & $\begin{array}{l}25.1 \pm \\
2.33\end{array}$ & $\begin{array}{l}24.9 \pm \\
2.94\end{array}$ & $\begin{array}{l}25.7 \pm \\
1.05\end{array}$ \\
\hline $\begin{array}{l}\text { BUN } \\
\text { (mg/dl) } \\
\text { After experiment. }\end{array}$ & $\begin{array}{l}25.1 \pm \\
2.5\end{array}$ & $\begin{array}{l}26.1 \pm \\
3.5\end{array}$ & $\begin{array}{l}25.1 \pm \\
2.4\end{array}$ & $\begin{array}{l}65.2 \pm \\
11.6\end{array}$ & $\begin{array}{l}32.9 \pm \\
2.9\end{array}$ & $\begin{array}{l}38.4 \pm \\
2.1\end{array}$ & $\begin{array}{l}27.0 \pm \\
3.1\end{array}$ \\
\hline$t$ & 0.86 & 0.8 & 0.2 & 10.8 & 4.1 & 3.1 & 1.29 \\
\hline $\mathbf{p}$ & $>0.05$ & $>0.05$ & $>0.05$ & $<0.01$ & $<0.05$ & $<0.05$ & $>0.05$ \\
\hline $\begin{array}{l}\text { Baseline creatinine clearance } \\
(\mathrm{ml} / \mathrm{min} / \mathbf{1 0 0 g})\end{array}$ & $\begin{array}{l}0.96 \pm \\
0.21\end{array}$ & $\begin{array}{l}0.99 \pm \\
0.18\end{array}$ & $\begin{array}{l}1.03 \pm \\
0.19\end{array}$ & $\begin{array}{l}0.97 \pm \\
0.17\end{array}$ & $\begin{array}{l}0.99 \pm \\
0.23\end{array}$ & $\begin{array}{l}0.98 \pm \\
0.13\end{array}$ & $\begin{array}{l}1.06 \pm \\
0.18\end{array}$ \\
\hline $\begin{array}{l}\text { Creatinine clearance after } \\
\text { experiment }\end{array}$ & $\begin{array}{l}0.93 \pm \\
0.21\end{array}$ & $\begin{array}{l}0.94 \pm \\
0.10\end{array}$ & $\begin{array}{l}1.05 \pm \\
0.22\end{array}$ & $\begin{array}{l}0.31 \pm \\
0.16\end{array}$ & $\begin{array}{l}0.76 \pm \\
0.12\end{array}$ & $\begin{array}{l}0.82 \pm \\
0.02\end{array}$ & $\begin{array}{l}0.92 \pm \\
0.16\end{array}$ \\
\hline$t$ & 0.31 & 0.76 & 0.22 & 8.9 & 2.4 & 3.4 & 1.8 \\
\hline $\mathbf{p}$ & $>0.05$ & $>0.05$ & $>0.05$ & $<0.01$ & $<0.05$ & $<0.05$ & $>0.05$ \\
\hline $\begin{array}{l}\text { Baseline } \\
\text { FENa }\end{array}$ & $\begin{array}{l}0.98 \pm \\
0.23\end{array}$ & $\begin{array}{l}1.03 \pm \\
0.19\end{array}$ & $\begin{array}{l}0.99 \pm \\
0.25\end{array}$ & $\begin{array}{l}0.97 \pm \\
0.07\end{array}$ & $\begin{array}{l}0.99 \pm \\
0.11\end{array}$ & $\begin{array}{l}1.04 \pm \bullet \\
0.10\end{array}$ & $\begin{array}{l}1.01 \pm \\
0.12\end{array}$ \\
\hline $\begin{array}{l}\text { FENa } \\
\text { after } \\
\text { experiment }\end{array}$ & $\begin{array}{l}0.99 \pm \\
0.13\end{array}$ & $\begin{array}{l}0.98 \pm \\
0.08\end{array}$ & $\begin{array}{l}1.01 \pm \\
0.22\end{array}$ & $\begin{array}{l}0.37 \pm \\
0.10\end{array}$ & $\begin{array}{l}0.80 \pm \\
0.08\end{array}$ & $\begin{array}{l}0.79 \pm \\
0.17\end{array}$ & $\begin{array}{l}0.98 \pm \\
0.27\end{array}$ \\
\hline $\mathbf{t}$ & 0.14 & 0.8 & 0.18 & 15.5 & 2.7 & 2.6 & 0.4 \\
\hline $\mathbf{p}$ & $>0.05$ & $>0.05$ & $>0.05$ & $<0.01$ & $<0.05$ & $<0.05$ & $>0.05$ \\
\hline
\end{tabular}

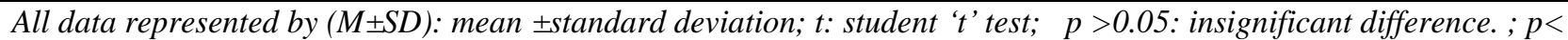
0.05: significant difference; $P<0.01$ : highly significant difference.

Table 4: ANOVA, One Way Statistical Analysis comparing after experiment mean values of serum creatinine, blood urea nitrogen (BUN), creatinine clearance and fractional excretion of sodium (FENa ${ }^{+}$) between group I: (control group); and group II: (received intravenous bicarbonate); group III: (received intra-peritoneal theophylline); group IV: (received intravenous urografin); group V: (intravenous bicarbonate three hours before urografin); group VI: (intra-peritoneal theophylline one hour before urografin); group VII: (bicarbonate three hours and theophylline one hour before urografin injection). (10 rats/group).

\begin{tabular}{|l|l|l|l|l|l|l|}
\hline parameters & $\begin{array}{l}\text { Group } \\
\text { I }\end{array}$ & $\begin{array}{l}\text { Group } \\
\text { IV }\end{array}$ & $\begin{array}{l}\text { Group } \\
\text { V }\end{array}$ & $\begin{array}{l}\text { Group } \\
\text { VI }\end{array}$ & $\begin{array}{l}\text { Group } \\
\text { VII }\end{array}$ & P \\
\hline $\begin{array}{l}\text { Serum Creatinine } \\
\text { (mg/dl) }\end{array}$ & $\begin{array}{l}0.46 \pm \\
\text { BUN (mg/dl) }\end{array}$ & $2.53 \pm 0.15^{*}$ & $\begin{array}{l}0.79 \\
\pm 0.25^{* \#}\end{array}$ & $0.67 \pm 0.23^{* \#}$ & $\begin{array}{l}0.47 \pm \\
0.04 \#\end{array}$ & $<\mathbf{0 . 0 1}$ \\
\hline $\begin{array}{l}\text { Creatinine clearance } \\
\text { (ml/min/100g) }\end{array}$ & $25.1 \pm 2.5$ & $\begin{array}{l}65.2 \pm \\
11.6^{*}\end{array}$ & $32.9 \pm 2.9^{* \#}$ & $38.4 \pm 2.1^{* \#}$ & $27.0 \pm 3.1 \#$ & $<\mathbf{0 . 0 1}$ \\
\hline FENa $^{+}$ & $0.93 \pm 0.21$ & $0.31 \pm .16^{*}$ & $0.76 \pm 0.12^{* \#}$ & $0.82 \pm 0.02 \#$ & $0.92 \pm 0.16 \#$ & $<\mathbf{0 . 0 1}$ \\
& $0.99 \pm$ & $0.37 \pm 0.10^{*}$ & $\begin{array}{l}0.80 \pm \\
0.08^{*} \#\end{array}$ & $\begin{array}{l}0.79 \pm \\
0.17 * \#\end{array}$ & $\begin{array}{l}0.98 \pm \\
0.27 \#\end{array}$ & $<\mathbf{0 . 0 1}$ \\
\hline
\end{tabular}

$P^{*}$ significant difference compared with group I, P\# significant difference compared with group IV; the data are given as Mean $\pm S D$ : mean \pm standard deviation. 
Table 5: Chi square test $\left(\mathrm{X}^{2}\right)$ comparing histological findings percentages (tubular necrosis, protein casts, medullary congestion and interstitial edema) between group I: (control group) and group IV: (received intravenous urografin); group V: (intravenous bicarbonate three hours before urografin); group VI: (intraperitoneal theophylline one hour before urografin); group VII: (bicarbonate three hours and theophylline one hour before urografin injection). (10 rats/group).

\begin{tabular}{|l|l|l|l|l|l|}
\hline & Group I & Group IV & Group V & Group VI & Group VII \\
\hline Tubular necrosis & $0 \%$ & $66 \% *$ & $31 \% * \#$ & $29 \% * \#$ & $1 \% \#$ \\
\hline protein casts & $0 \%$ & $56 \% *$ & $33 \% * \#$ & $25 \% * \#$ & $0 \% \#$ \\
\hline Medullary congestion & $0 \%$ & $78 \% *$ & $23 \% * \#$ & $20 \% * \#$ & $2 \% \#$ \\
\hline Interstitial edema & $0 \%$ & $70 \% *$ & $27 \% * \#$ & $12 \% * \#$ & $3 \% \#$ \\
\hline
\end{tabular}

*P significant difference compared to control. \#P significant difference compared to group IV.

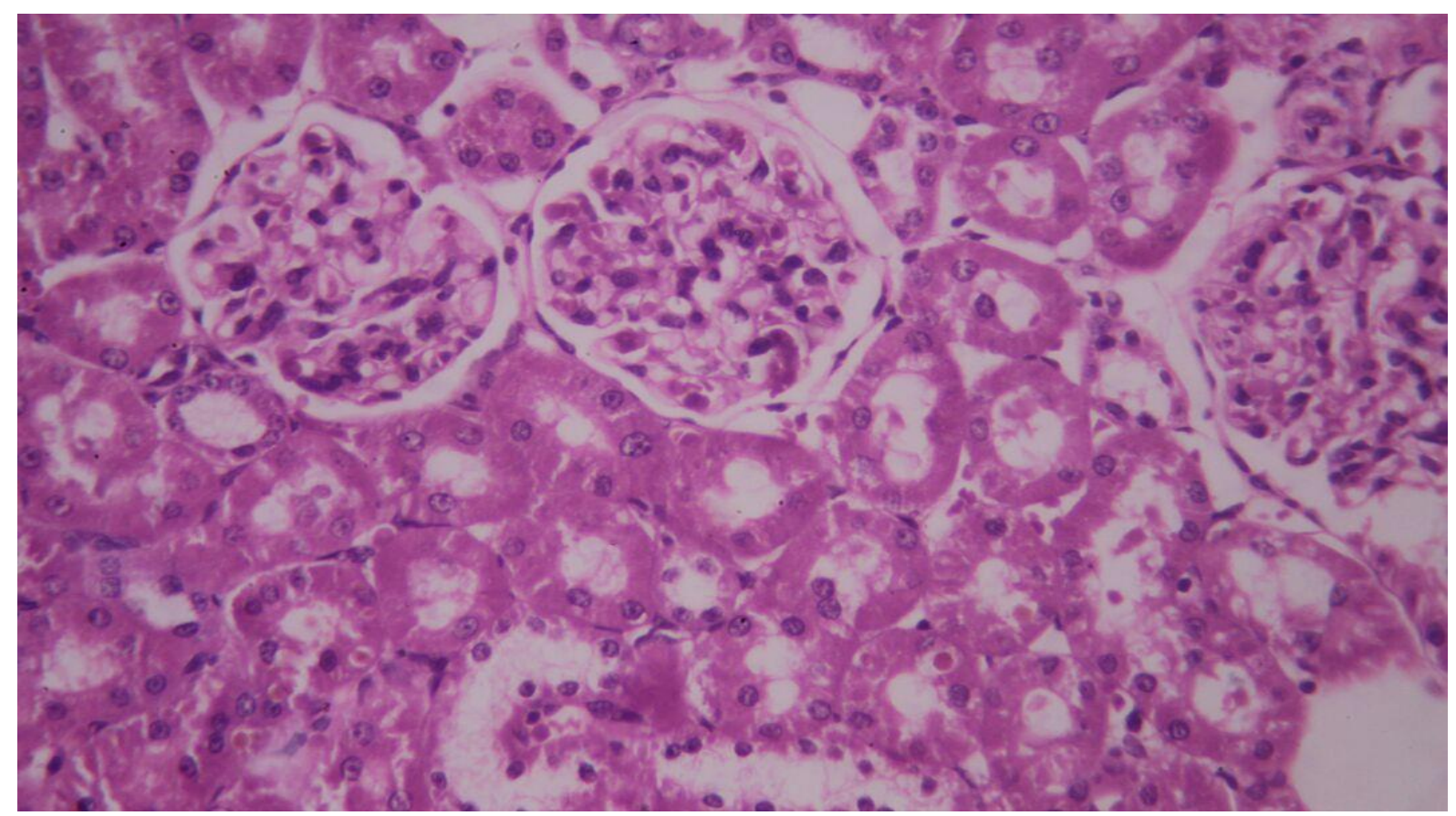

Figure 1: A photomicrograph of kidney tissue section of control rat showing the typical histological structure of renal glomerulus ( ) surrounded by Bowman's space ( ). Proximal convoluted tubules ( ) lined by pyramidal cells with indistinct cell boundaries and deeply acidophilic cytoplasm. The distal convoluted tubules ( ) are lined with cuboidal cells and have less acidophilic cytoplasm and a wider lumen. (H \& E X400). 


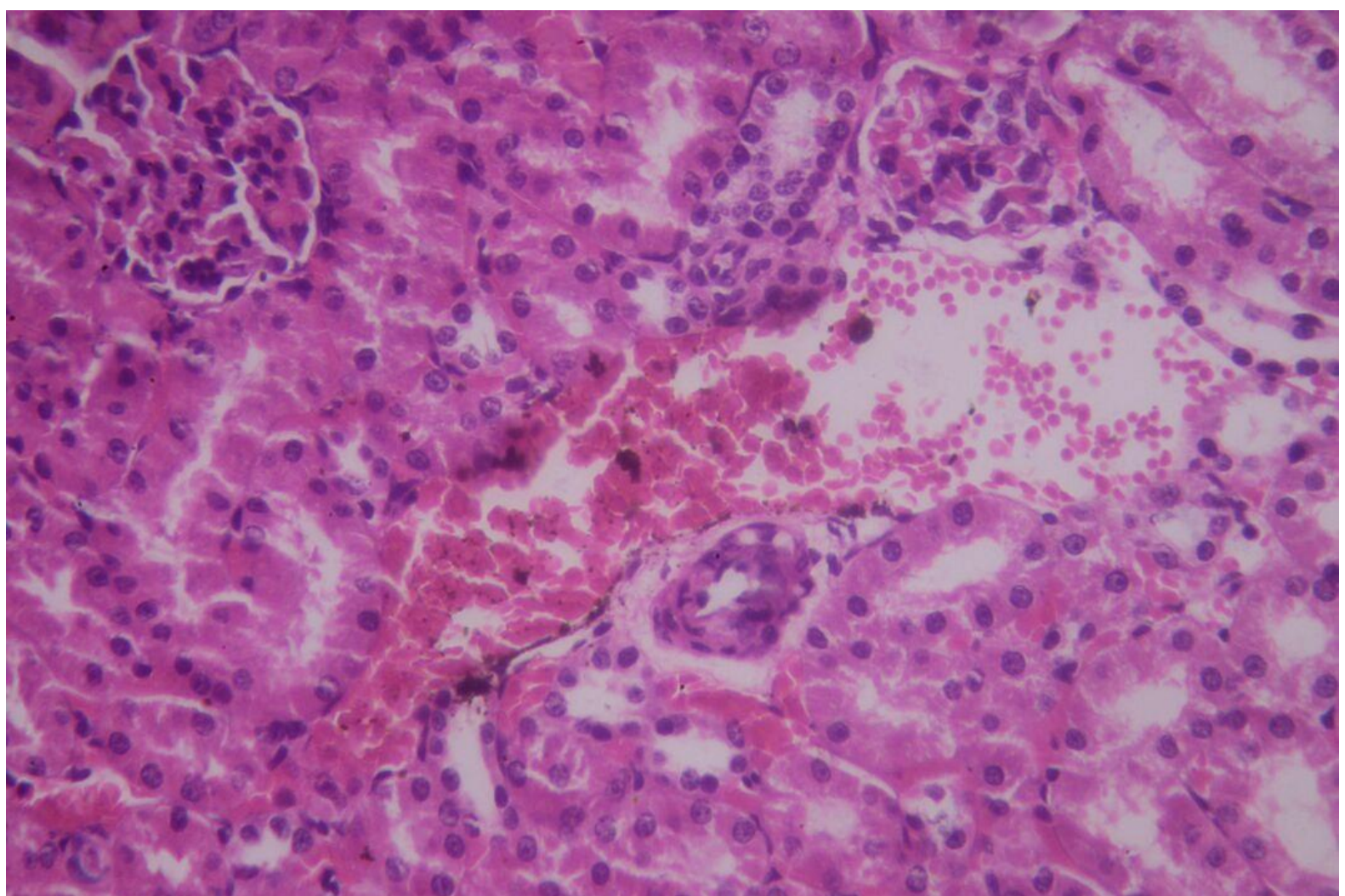

Figure 2: A photomicrograph of kidney tissue section from rats received urografin (group IV) revealed irregular glomerulus, desquamated Bowman's capsule, tubular dilatation and necrosis ( ), desquamated and vacuolated tubules ( ), peri tubular congestion ( ) and extravasations of blood between tubules. (H \& E X400).

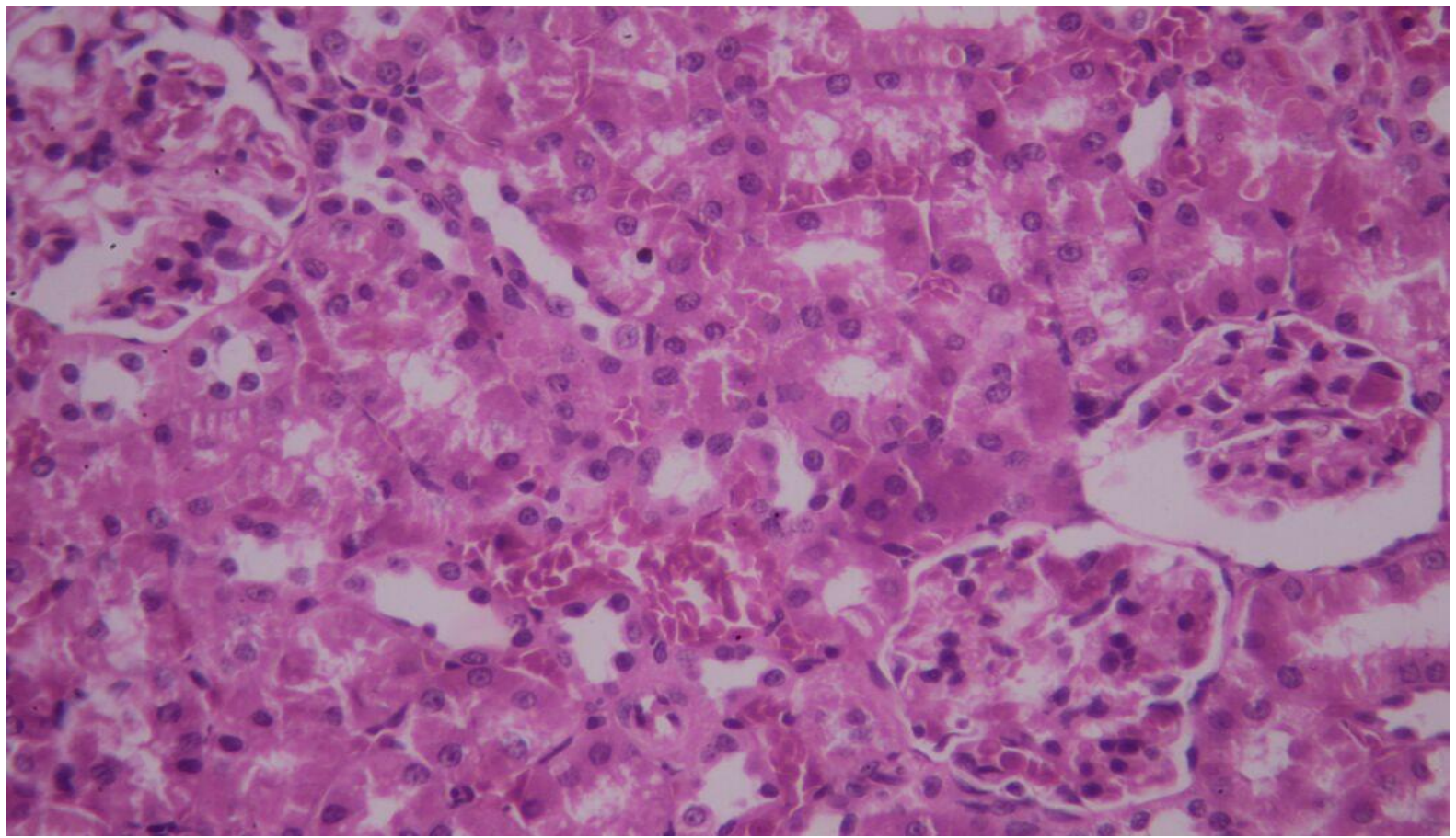

Figure 3: A photomicrograph of kidney tissue section of rat received intravenous bicarbonate three hours before urografin (group V), showed peri-tubular capillary congestion ( $\quad$ ) (H \& E X400). 


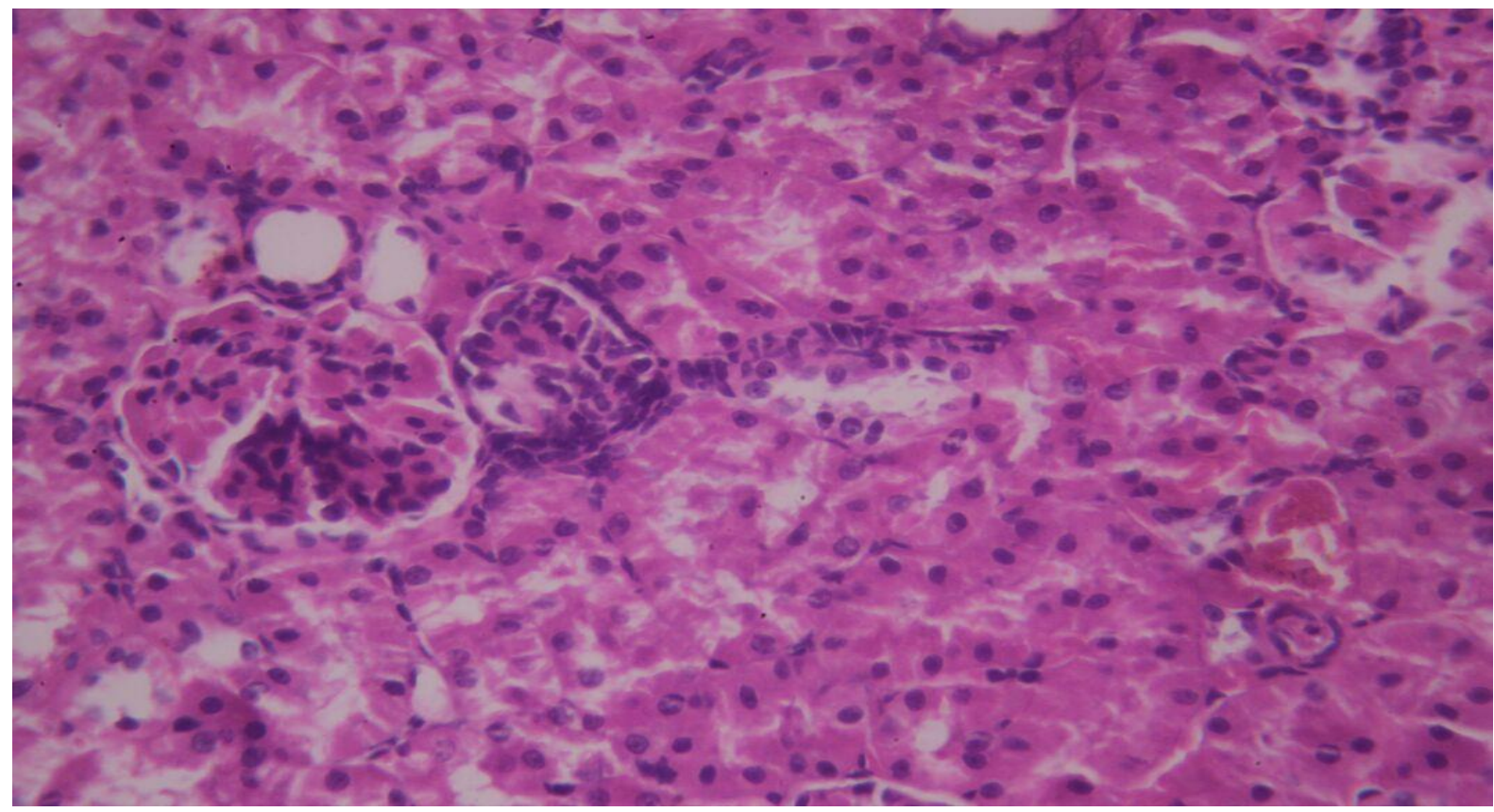

Figure 4: A photomicrograph of kidney tissue section of rat received intra-peritoneal theophylline one hour before urografin (group VI) showed, protein cast ( ), peri-tubular capillary congestion ( ). (H \& E X 400).

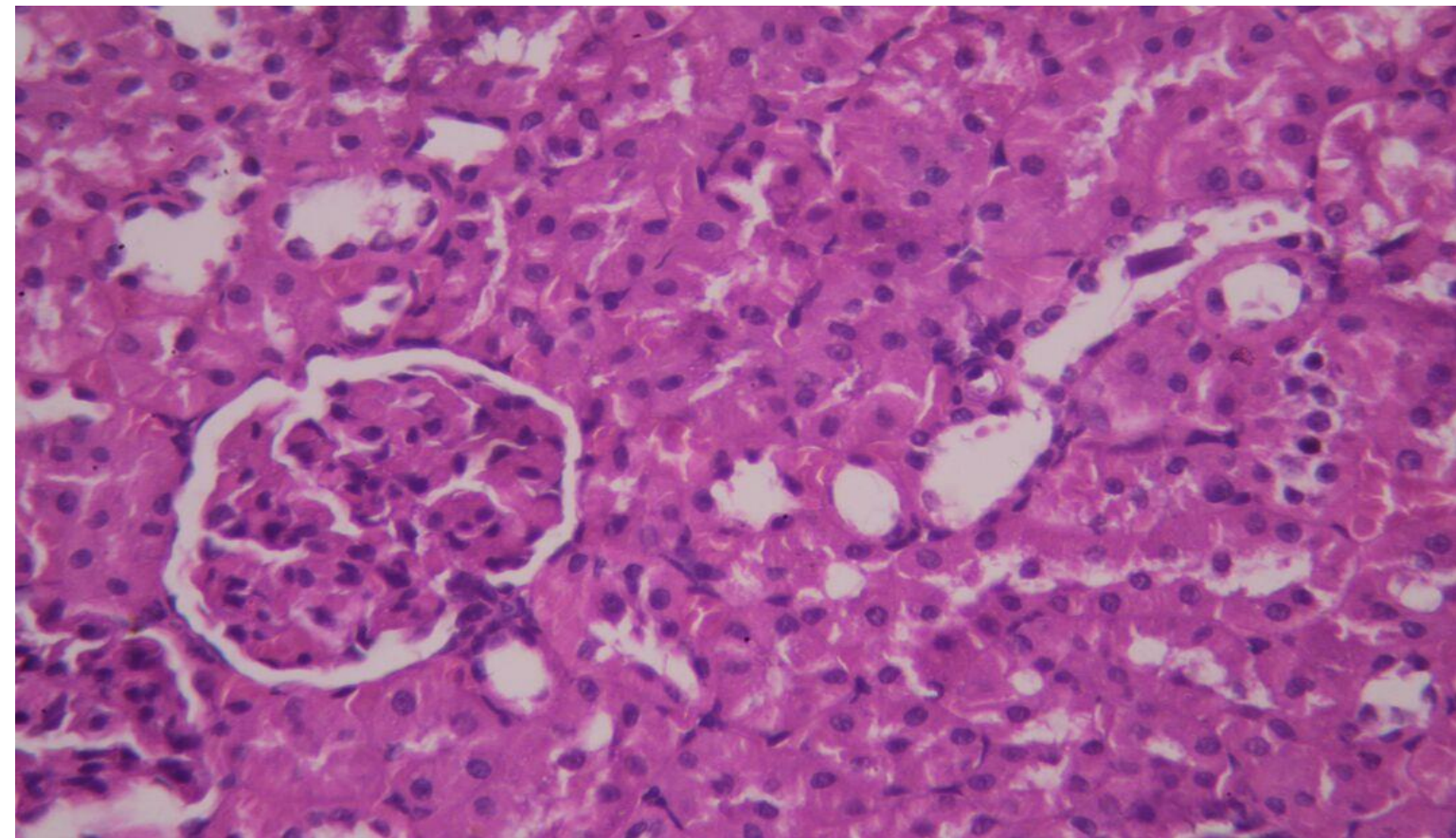

\section{Figure 5: A photomicrograph of kidney tissue section of rat received bicarbonate three hours and theophylline one hour before urografin injection (group VII) showed near normal kidney structure. (H} \& E X 400).

\section{Discussion}

CIN is generally regarded as a decline in renal function after contrast procedures. It cannot be regarded as a benign complication as it accounts for $12 \%$ of all cases of acute renal failure (Yang etal.,2014; Zhang et al.,2015), particularly in high risk patients, such as patients with chronic kidney disease, marked volume depletion, severe heart failure, diabetes or multiple contrast studies within 72-hours (Hörl,2009). As its risk factors are well characterized and the timing of renal insult is commonly known in advance, so it may be preventable. Previously, in low risk patients intravenous volume expansion is probably sufficient, however, in high risk patients additional prophylactic measures are needed, but their efficacy is not clearly defined (Weisbord et al.,2013). Various interventions were evaluated for the prevention of CIN, the most studied medications were theophylline or sodium bicarbonate long term infusion, with controversy on their efficacy (Arabmomeni et al., 2015; Lefel et al., 2016). So the present study was aimed to compare the 
efficacy of short-term infusion regimens of sodium bicarbonate, theophylline and the combination of both for prevention of contrast-induced nephropathy in rats.

In the present study, rats injected with urografin showed development of CIN both by laboratory and histopathological findings. There was more than $25 \%$ increase in the mean values of serum creatinine, serum BUN and significant decrease in the creatinine clearance and fractional excretion of sodium percentage when post experiment levels compared with the baseline levels and to control group. Histopathologically there was severe damage consisting of tubular necrosis, protein cast, medullary congestion and interstitial edema. These results were in accordance with the previous studies of Toprak et al.(2008) and Barlak et al. (2010) where there was an intermediate-severe injury in the radio-contrast group of rats.

Contrast nephropathy obtained in this study by using ionic hyper-osmolar contrast dye (urografin) and adequate dehydration as risk factors. It has been shown that dehydration potentiates the vasoconstrictive effects of contrast dye (Toprak et al., 2008).

The most widely used CIN definition is from the Contrast Media Safety Committee of the European Society of Urogenital Radiology; is an increase in serum creatinine equal to or more than $25 \%$ of baseline values or an absolute rise of $0.5-1.0 \mathrm{mg} / \mathrm{dL}$ in 48 hours after injection of a contrast agent (Mahmoodi etal.,2014; Lefel et al.,2016). Renal insufficiency was usually defined as a decrease in glomerular filtration rate (GFR), and since the GFR has to fall by $50 \%$ before a rise in serum creatinine occurs, an elevated serum creatinine level was used as the cut-off point for the definition of renal insufficiency (Klima et al., 2012).

The

low fractional excretion of sodium $\left(\mathrm{FENa}^{+}\right)$raise the possibility that acute renal failure secondary to contrast media may be mediated either by decrease in renal perfusion or by acute tubular obstruction by protein casts that is associated with CIN (Legrand et al.,2016).

Although the pathophysiology of CIN is complex and partially understood, some experimental studies show evidence of acute tubular necrosis. This is caused by three different but interacting pathways: the haemo-dynamic effects of contrast dye, the effect of reactive oxygen species (ROS) and direct contrast dye tubular cell toxicity (Barlak et al., 2010).

Hypoxic medullary injury plays a critical role in the pathogenesis (Heyman et al., 2008). The mechanism for medullary hypoxia is a combination of a decline in local micro-circulatory blood flow and increased oxygen demand of tubular cells (Persson et al., 2005).
Firstly, the decline in local blood flow has been resulted from an imbalance that occurs between vasoconstrictive (vasopressin, adenosine, angiotensin II and endothelin) and vasodilatative mediators (dopamine, nitric oxide, atrial natriuretic peptide and prostaglandin E2) in response of contrast dye administration (Heyman et al., 2005). Furthermore, the distribution of mediator's receptor subtypes in the cortex and medulla may be responsible for different regional haemo-dynamic responses (Heyman et al.,2008). The medullary hypoxia induces generation of ROS which, once exceeding the cellular scavenging capacities, they lead to mitochondrial, nuclear DNA, membrane lipids and cellular proteins injury (Heyman et al., 2010; Abouzeid and Mosbah, 2016). In addition, the ROS increase endothelin and angiotensin-II induced vasoconstriction and reduces the bioavailability of the vasodilatative nitric oxide leading to increased tone and reactivity of afferent arterioles (Haller and Hizoh, 2004).

Secondly, increased oxygen consumption of tubular cells, caused by the osmotic load generated by injected contrast dye and the endothelin release, leads to more active re-absorption of sodium by distal tubular cells (Heyman et al., 2008).

In the present study, bicarbonate administration before urografin ameliorated the elevated serum creatinine, BUN, the decrease in the creatinine clearance and fractional excretion of sodium percentage but they still show significant difference when compared with the control group. In addition, the examined kidney specimens showed mild tubular necrosis, protein cast, medullary congestion and interstitial edema. The dose of sodium bicarbonate used in this study was determined on the basis of studies that demonstrated this concentration to be effective levels for the decrease in lipid peroxidation and oxidative renal injury in experimental animal models (Barlak et al., 2010).

These results were in accordance with the previous experimental study of Barlak et al. (2010), who found that hypertonic sodium bicarbonate attenuates the development of radio-contrast-induced tubular necrosis, but there was no significant effect on serum creatinine and creatinine clearance levels. Multiple previous clinical trials investigated the use of isotonic sodium bicarbonate infusion before ionic low osmolar contrast media, and they revealed that it reduces the incidence of CIN when compared to hydration with isotonic fluids strategy. Moreover, they concluded that it is the treatment of choice in the prevention of CIN, because of less prescribed fluid volume and a lesser time required for infusion of the fluid (Merten et al.,2004; Mahmoodi etal.,2014; Yeganehkhah et al.,2014).

Some studies showed that long term regimen of bicarbonate infusion was a more effective strategy to prevent CIN than the short term regimen (Kama et al., 2014 ;Abouzeid and Mosbah ,2016). On the other hand, other studies found that the long and the short 
term regimens have the same effectiveness with very high safety, even in patients with heart failure and thus, it may be the regimen of choice because it is very easy to apply, even to outpatient procedures (Mueller , 2006; Briguori et al., 2007; Zoungas et al.,2009). In line with these results, a more pronounced efficacy of sodium bicarbonate was noted in patients undergoing emergency procedures compared with those undergoing elective procedures (Masuda et al.,2007; Ueda etal.,2011).

On the contrary, some studies have indicated that the effectiveness of isotonic sodium bicarbonate treatment to prevent $\mathrm{CIN}$ in high risk patients, remains uncertain and secondary clinical endpoints as renal replacement therapy and mortality were not improved, hence sodium bicarbonate prophylaxis guidelines cannot be generalized to a heterogeneous ICU population (Gomes et al.,2012; Hafiz et al.,2012, Kristeller et al.,2013; Boucek et al.,2013; Inda-Filho et al.,2014; Zhang et al.,2015; Solomon et al.,2015;Lefel et al.,2016). In addition, volume supplementation with isotonic saline was found to be superior to sodium bicarbonate for the prevention of CIN (Klima et al., 2012; Schiffl, 2015). Furthermore, a previous retrospective cohort study concluded that sodium bicarbonate was associated with an increased incidence of CIN (From et al., 2008).

Lefel et al.(2016) explained these findings as, prophylactic bicarbonate therapy addresses only contrast dye administration, which is only one possible risk factor for the development of renal dysfunction, while ICU patients frequently are exposed to multiple potential risk factors for renal impairment as; heart failure, anaemia, sepsis, hypoxia and medications which can all negatively affect kidney function. Furthermore, Klima et al. (2012) explained these findings by the early termination of studies, publication bias, small differences in the concentration, the overall amount of sodium bicarbonate applied, the type of the contrast procedure and the patient selection.

Various mechanisms have been proposed to explain how sodium bicarbonate administration prevents CIN. One suggestion is that, oxygen free radicals and peroxide are usually generated in acidic conditions, and sodium bicarbonate makes tubular urine more alkaline, subsequently it could reduce the production of free radicals and peroxide (McCullough et al., 2006; Burgess and Walker,2014). In addition, Mueller (2006) stated that the role of bicarbonate is attributed to buffering of acidosis-induced vasoconstriction, which may amplify the vasoconstriction induced by the contrast agent.

The present study has demonstrated that theophylline administration before urografin ameliorated the elevated serum creatinine, BUN, the decrease in the creatinine clearance and fractional excretion of sodium percentage but they still show significant difference when compared with the control group. Histopathologically, the examined kidney sections showed mild damage consisting of tubular necrosis, protein cast, medullary congestion and interstitial edema.

It has been shown that theophylline, non selective adenosine antagonist, prevents CIN in both animal models and humans. Deray et al.(1990) and Erley et al.(1994) studies found a significant decrease in GFR, after non-ionic low osmolar contrast media (iopromide) injection, while the GFR remained constant in the group of patients receiving theophylline. In addition, Arakawa et al. (1996) demonstrated that both theophylline and adenosine $\left(\mathrm{A}_{1}\right)$ selective receptor antagonists markedly prevent contrast media induced deterioration in renal function in dogs with renal insufficiency. Furthermore, Erley et al. (1997) study in nitric oxide depleted rats, showed a significant increase of the renal vasoconstrictive effect of contrast media, while theophylline pre-treated rats showed complete protection against the decline of renal blood flow and GFR induced by the contrast media. Kolonko et al. (1998) stated that highosmolar contrast medium induced impairment of renal excretory, endocrine and tubular function could be prevented by giving pre-treatment with theophylline. The study of Oldroyd et al. (2000) found that urografin produced a fall in GFR and renal perfusate flow (RPF) and theophylline prevented the fall in GFR but did not affect the decreases in RPF. They suggested a role for adenosine acting at the $A_{1}$ receptor in mediating the decrease in GFR induced by contrast dye. Previously, Forty one controlled trials found that theophylline reduced the risk for CIN more than saline. However, there is lack of a statistically significant reno-protective effect which may result from insufficient data or study heterogeneity (Kelly et al., 2008). In addition it was found that, theophylline given intravenously 30 minutes before contrast media injection is renoprotective, particularly in intensive care unit patients (Hörl , 2009). A previous meta-analysis conducted by Dai et al. (2012) on sixteen randomized controlled trials assessing adenosine antagonists for prevention of CIN showed that theophylline significantly decreased the risk of CIN in thirteen trials and had a protective effect on the absolute change in serum creatinine concentration. However, beneficial effects of theophylline were not observed in patients with high baseline creatinine values, In addition, its long-term effect on risk of dialysis and in-hospital mortality was not established. Furthermore, Kwok et al. (2013) revealed that theophylline interventions significantly decreased the risk for CIN but the limitations in the study quality and heterogeneity preclude any firm recommendations. A randomized controlled trial found that theophylline is superior to $\mathrm{N}$-acetylcysteine in preventing CIN in patients, however they recommend further trials with larger sample of patients (Arabmomeni et al., 2015).

On the contrary, Erley et al. (1999) investigated the effect of the oral administration of theophylline on changes in renal haemodynamics and tubular injury induced by contrast dye in well-hydrated 
patients with mild-to-moderate renal insufficiency. Their results indicate that GFR is preserved by hydration alone and the theophylline did not bring any additional benefit. They recommend the usage of theophylline in patients where sufficient hydration may be impossible or in patients with a concomitant decrease in renal blood flow (e.g. congestive heart failure).

Adenosine, is present in the cytosol as well as at extracellular sites of the kidney. Extracellular adenosine acts on adenosine receptor subtypes $\left(\mathrm{A}_{1}, \mathrm{~A}_{2}\right.$ and $\left.A_{3}\right)$ in the cell membranes to affect vascular and tubular functions. During oxygen deficiency or during increased tubular transport work the rate of adenosine formation is enhanced. Experiments on laboratory animals clearly show that it acts as a vaso-constrictive metabolite in the kidney so decreases GFR by constricting afferent arterioles via $A_{1}$ receptors, especially in superficial nephrons, and thus lowers the salt load and transport work of the kidney (Jenik et al., 2000; Oldroyd et al., 2000 ; Osswald et al.,1995). On contrary, it leads to vasodilation via $\mathrm{A}_{2}$ receptors in the deep cortex and exerts differential effects on sodium chloride transport along the tubular and collecting duct system (Arakawa et al., 1996 ;Vallon and Osswald, 2009). These vascular and tubular effects point to the important role of adenosine and its receptors in the intra-renal metabolic regulation of kidney function, which form the basis for potential therapeutic approaches in CIN (Osswald et al., 1995).

The detrimental effects of contrast dye are mediated partially by the effect of intra-renal formed adenosine on $A_{1}$ receptors which are involved in the development of contrast induced renal vasoconstriction, and may lead to acute renal failure (Kolonko etal.,1998). The renal protective effects of theophylline are mediated by the blockade of adenosine action and prevented the renal deterioration (Arakawa et al., 1996; Erley et al.,1999 ).

In the present study, combination administration of bicarbonate three hours and theophylline one hour before urografin injection showed insignificant difference in all laboratory parameters when compared with the control group. Furthermore, the histopathological examination of kidney sections showed marked improvement of all changes as regards tubular necrosis, protein cast, medullary congestion and interstitial edema.

\section{Conclusion}

The present study showed that urografin injection resulted in marked nephrotoxicity manifested biochemically and histopathologically. Pre-treatment with sodium bicarbonate or theophylline partially ameliorated the CIN. While, combined administration of sodium bicarbonate and theophylline before urografin injection showed marked improvement of renal function and histopathological examination in rats.

\section{Recommendations}

With the further increase of investigations using contrast media and co morbidities of the patients, one may suggest that the problem of contrast media induced nephropathy will further increase, despite all prophylactic procedures so far recommended. Thus, the combination therapy with sodium bicarbonate and theophylline before urografin injection is recommended in order to allow safe methods. This might be of special importance for all emergency cases or the high-risk patients, where sufficient hydration may be impossible. In addition, further clinical studies are needed.

\section{Acknowledgement}

We gratefully acknowledge Dr. Mona Hussein Raafat, associate professor of histology, Faculty of medicine, Ain shams university, for performing the histological work for this study.

\section{References}

Abouzeid S and Mosbah O (2016): Evaluation of different sodium bicarbonate regimens for the prevention of contrast medium-induced nephropathy. Electron Physician. Feb 25;8(2):1973-7.

Arabmomeni M, Najafian J, Abdar Esfahani M et al., (2015): Comparison between theophylline, Nacetylcysteine, and theophylline plus Nacetylcysteine for the prevention of contrastinduced nephropathy. ARYA Atheroscler. Jan;11(1):43-9.

Arakawa K, Suzuki H, Naitoh M et al., (1996): Role of adenosine in the renal responses to contrast medium. Kidney Int. May;49(5):1199-206.

Au TH, Bruckner A, Mohiuddin SM et al., (2014): The prevention of contrast induced nephropathy. Ann Pharmaco ther. 48:1332-42.

Barlak A, Akar H, Yenicerioglu Y et al., (2010): Effect of sodium bicarbonate in an experimental model of radio-contrast nephropathy. Ren Fail. ;32(8):992-9.

Boucek P, Havrdova T, Oliyarnyk O et al., (2013): Prevention of contrast induce nephropathy in diabetic patients with impaired renal function: a randomized, double blind trial of sodium bicarbonate versus sodium chloride-based hydration. Diabetes Res Clin Pract. Sep;101(3):303-8.

Briguori C, Airoldi F, D’Andrea D et al., (2007): Renal Insufficiency Following Contrast Media Administration Trial (REMEDIAL) a randomized comparison of 3 preventive strategies. Circulation;115:1211-17.

Burgess WP and Walker PJ (2014): Mechanisms of contrast-induced nephropathy reduction for 
saline $(\mathrm{NaCl})$ and sodium bicarbonate (NaHCO3). Biomed Res Int; 2014:510385.

Dai B, Liu Y, Fu L et al.,(2012): Effect of theophylline on prevention of contrast-induced acute kidney injury: a meta-analysis of randomized controlled trials. Am J Kidney Dis. Sep;60 (3):360-70.

Deray G, Martinez F, Cacoub P et al.,(1990):A role for adenosine calcium and ischemia in radiocontrast induced intra-renal vasoconstriction. Am J Nephrol 10:316-322.

Erley Cm, Duda Sh, Schlepckow S et al., (1994): Adenosine antagonist theophylline prevents the reduction of glomerular filtration rate after contrast media application. Kidney $\mathrm{mt}$ 45:1425-1431.

Erley CM, Duda SH, Rehfuss D et al., (1999): Prevention of radio-contrast-media-induced nephropathy in patients with preexisting renal insufficiency by hydration in combination with the adenosine antagonist theophylline. Nephrol Dial Transplant. May; 14(5):1146-9.

Erley CM, Heyne N, Burgert K etal., (1997): Prevention of radio-contrast induced nephropathy by adenosine antagonists in rats with chronic nitric oxide deficiency. $\mathrm{J}$ Am Soc Nephrol. Jul;8(7):1125-32.

From AM, Bartholmai BJ, Williams AW et al., (2008): Sodium bicarbonate is associated with an increased incidence of contrast nephropathy: a retrospective cohort study of 7977 patients at mayo clinic. Clin J Am Soc Nephrol. Jan; 3(1):10-8.

Gomes VO, Lasevitch R, Lima VC et al., (2012): Hydration with sodium bicarbonate does not prevent contrast nephropathy: a multicenter clinical trial. Arq Bras Cardiol;99:1129-34.

Hafeez AR, Idrees MK and Akhtar SF (2016): Accuracy of GFR estimation formula in determination of glomerular filtration rate in kidney donors: Comparison with $24 \mathrm{~h}$ urine creatinine clearance. Saudi J Kidney Dis Transpl. Mar;27(2):320-5.

Hafiz AM, Jan MF, Mori N et al., (2012): Prevention of contrast-induced acute kidney injury in patients with stable chronic renal disease undergoing elective per-cutaneous coronary and peripheral interventions: Randomized comparison of two preventive strategies. Catheter Cardio-vasc Interv;79:929-37.

Haller C and Hizoh I (2004): The cyto-toxicity of iodinated radio-contrast agents on renal cells in vitro. Invest Radiol 39:149-154.

Heyman SN, Rosen S and Rosenberger C (2008): Renal parenchymal hypoxia, hypoxia adaptation and the pathogenesis of radio- contrast nephropathy. Clin J Am Soc Nephrol $3: 288-296$

Heyman SN, Rosen S, Khamaisi M et al., (2010): Reactive oxygen species and the pathogenesis of radio-contrast induced nephropathy. Invest Radiol 45:188-195.

Heyman SN, Rosenberger C and Rosen S (2005): Regional alterations in renal hemodynamcs and oxygenation: a role in contrast medium induced nephropathy. Nephrol Dial Transplant 20 (Suppl 1):i6-i11.

Hörl WH (2009): [Contrast induced nephropathy]. Wien Klin Wochenschr. ;121(1-2):15-32.

Inda-Filho AJ, Caixeta A, Manggini M etal., (2014): Do intravenous $\mathrm{N}$-acetylcysteine and sodium bicarbonate prevent high osmolal contrast-induced acute kidney injury? A randomized controlled trial. PLoS One. Sep 25;9(9):e107602.

Jenik AG, Ceriani Cernadas JM, Gorenstein A et al., (2000): A randomized, double-blind, placebocontrolled trial of the effects of prophylactic theophylline on renal function in term neonates with perinatal asphyxia. Pediatrics. Apr;105(4):E45.

Kama A, Y1lmaz S, Yaka E et al., (2014 ): Comparison of short-term infusion regimens of $\mathrm{N}$ acetylcysteine plus intravenous fluids, sodium bicarbonate plus intravenous fluids, and intravenous fluids alone for prevention of contrast-induced nephropathy in the emergency department. Acad Emerg Med. Jun; 21(6):615-22.

Kelly AM, Dwamena B, Cronin P etal., (2008): Metaanalysis: effectiveness of drugs for preventing contrast-induced nephropathy. Ann Intern Med. Feb 19;148(4):284-94.

Klima T, Christ A, Marana I et al.,(2012): Sodium chloride vs. Sodium bicarbonate for the prevention of contrast medium-induced nephropathy: a randomized controlled trial. Eur Heart J;33:2071-9.

Kolonko A, Wiecek A and Kokot F(1998): The non selective adenosine antagonist theophylline does prevent renal dysfunction induced by radiographic contrast agents. J Nephrol. MayJun;11(3):151-6.

Kristeller JL, Zavorsky GS, Prior JE et al.,(2013): Lack of effectiveness of sodium bicarbonate in preventing kidney injury in patients undergoing cardiac surgery: a randomized controlled trial. Pharmacotherapy; 33:710-17.

Kwok CS, Pang CL, Yeong JK et al., (2013): Measures used to treat contrast-induced nephropathy: overview of reviews. $\mathrm{Br} \mathrm{J}$ Radiol. Jan;86(1021):20120272. 
Lawrence M and Robert HC (1993): Methods of determination of blood urea nitrogen and serum creatinine, In :Tietz textbook of clinical chemistry, $2^{\text {nd }}$ edition, WB Saunders Company ,London, pp.621.

Lefel N, Janssen L, le Noble J et al., (2016): Sodium bicarbonate prophylactic therapy in the prevention of contrastinduced nephropathy in patients admitted to the intensive care unit of a teaching hospital: a retrospective cohort study. J Intensive Care. Jan 12;4:5.

Legrand M, Le Cam B, Perbet S et al., (2016): Urine sodium concentration to predict fluid responsiveness in oliguric ICU patients: a prospective multicenter observational study. Crit Care. May 29;20(1):165.

Mahmoodi K, Sohrabi B, Ilkhchooyi F, et al., (2014): The Efficacy of Hydration with Normal Saline Versus Hydration with Sodium Bicarbonate in the Prevention of Contrastinduced Nephropathy. Heart Views. Apr;15(2):33-6.

Masuda M, Yamada T, Mine T et al.,(2007): Comparison of usefulness of sodium bicarbonate versus sodium chloride to prevent contrast-induced nephropathy in patients undergoing an emergent coronary procedure. Am J Cardiol;100:781-6.

McCullough PA, Adam A, Becker CR et al., (2006): Risk prediction of contrast-induced nephropathy. Am J Cardiol;98:27-36.

Merten GJ, Burgess WP, Gray LV et al., (2004): Prevention of contrast-induced nephropathy with sodium bicarbonate: a randomized controlled trial. JAMA. May 19;291(19):2328-34.

Mueller C (2006): Prevention of contrast-induced nephropathy with volume supplementation. Kidney Int Suppl. 2006; 69: S16-19.

Oldroyd SD, Fang L, Haylor JL et al., (2000): Effects of adenosine receptor antagonists on the responses to contrast media in the isolated rat kidney. Clin Sci (Lond). Mar;98(3):303-11.

Osswald H, Gleiter C and Mühlbauer B (1995): Therapeutic use of theophylline to antagonize renal effects of adenosine. Clin Nephrol. Jan;43 Suppl 1:S33-7.

Ozturk SA, Ceylan C, Serel TA et al., (2015): Protective effect of theophylline on renal functions in experimental pneumo-peritoneum model. Ren Fail. May 11:1-6.

Persson PB, Hansell P and Liss P (2005): Pathophysiology of contrast medium-induced nephropathy. Kidney International 68:14-22.
Rudnick M and Feldman H (2008): Contrast-induced nephropathy: What are the true clinical consequences? Clin J Am Soc Nephrol.; 3(1):263-272.

Schiffl H (2015): Sodium bicarbonate infusion for prevention of acute kidney injury: no evidence for superior benefit, but risk for harm? Int Urol Nephrol. Feb;47(2):321-6.

Solomon R, Gordon P, Manoukian SV et al.,( 2015): Randomized Trial of Bicarbonate or Saline Study for the Prevention of ContrastInduced Nephropathy in Patients with CKD. Clin J Am Soc Nephrol. Sep 4;10(9):1519-24.

Taylor JK (1990): statistical technique for data analysis. $2^{\text {nd }}$ ed. Lewis Pub. Inc. USA. P.2530.

Toprak O, Cirit M, Tanrisev M et al., (2008): Preventive effect of nebivolol on contrastinduced nephropathy in rats. Nephrol Dial Transplant; 23(3):853-9.

Ueda H, Yamada T, Masuda M et al.,(2011): Prevention of contrast-induced nephropathy by bolus injection of sodium bicarbonate in patients with chronic kidney disease undergoing emergent coronary procedures. Am J Cardiol;107:1163-7.

Vallon V and Osswald H (2009): Adenosine receptors and the kidney. Hand b Exp Pharmacol. ;(193):443-70.

Weisbord SD, Gallagher M, Kaufman J et al., (2013): Prevention of contrast-induced AKI: a review of published trials and the design of the prevention of serious adverse events following angiography (PRESERVE) trial. Clin J Am Soc Nephrol. Sep;8 (9):1618-31.

Yang K, Liu W, Ren W et al., (2014): Different interventions in preventing contrast-induced nephropathy after percutaneous coronary intervention. Int Urol Nephrol;46:1801-7.

Yeganehkhah MR, Iranirad L, Dorri F et al., (2014): Comparison between three supportive treatments for prevention of contrastinduced nephropathy in high-risk patients undergoing coronary angiography. Saudi J Kidney Dis Transpl. Nov;25(6):1217-23.

Zhang B, Liang L, Chen W et al., (2015): The efficacy of sodium bicarbonate in preventing contrastinduced nephropathy in patients with preexisting renal insufficiency: a meta-analysis. BMJ Open. Mar 17; 5(3): e006989.

Zoungas S, Ninomiya T, Huxley R et al., (2009): Systematic review: sodium bicarbonate treatment regimens for the prevention of contrast-induced nephropathy. Ann Intern Med.; 151: 631-8. 


\section{الملخص العربي}

التأثير الوقائي لنظام الضخ علي المدي القصيربيكربونات الصوديوم و الثيوفيللين ضد الفشل الكلوي المستحث من صبغه اليوروجرافيين فى الفئران

\section{جيهان بشري عزب و رباب نبيل حافظا}

الفشل الكلوي الناجم عن استخدام الصبغات يعتبر حاله شائعه مصحوبه بنتائج خطيره و التي.من الممكن بتنبها حيث ان العوامل

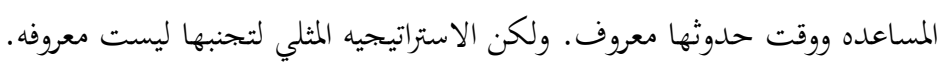

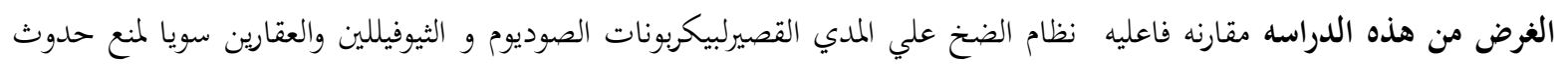
الفشل الكلوي الناتج عن حقن صبغه اليوروجرافيين فن الفئران. الطرق:تم اجراء هذه الدراسه علي سبعين من ذكور الجرذان البيضاء. تم تقسيمها إلى سبعه بحموعات. البحموعة الأولى (بحموعة ضابطه)،

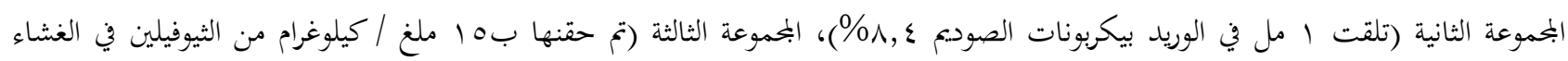

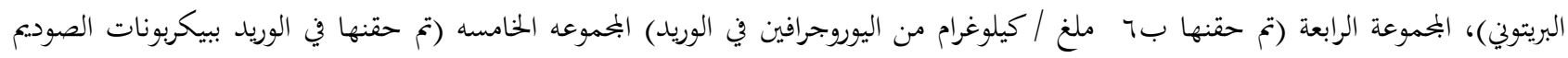

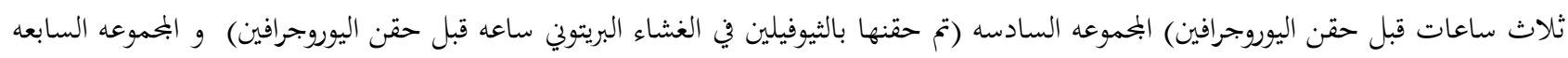

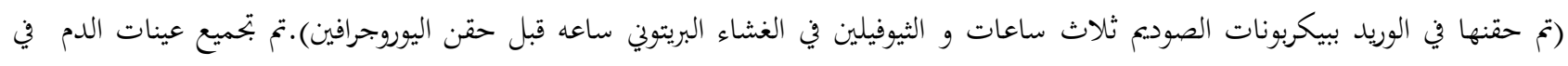

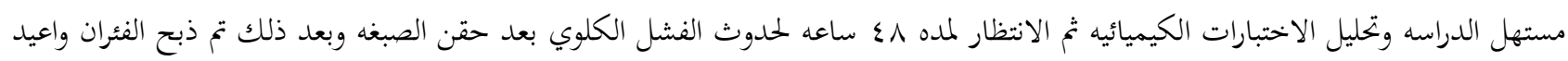

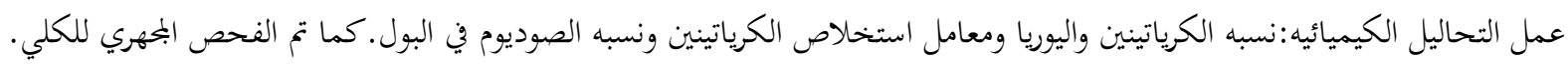

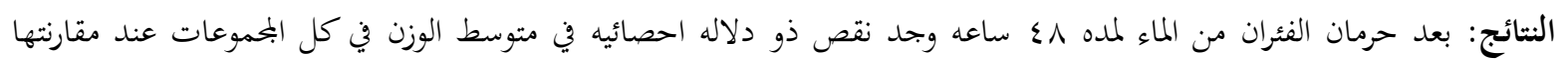

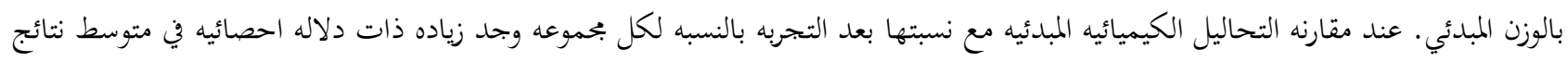
نسبه الكرياتينين والبولينا و نقص ذو دلاله احصائيه في نسبه معامل استخلاص الكرياتينين ونسبه الصوديوم في البول في المحموعات الرابعه والخامسه فئه

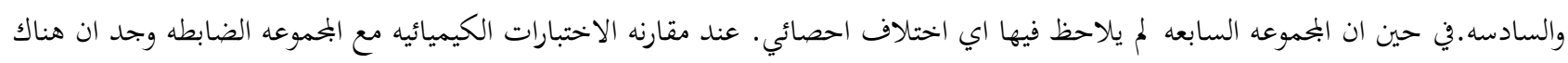

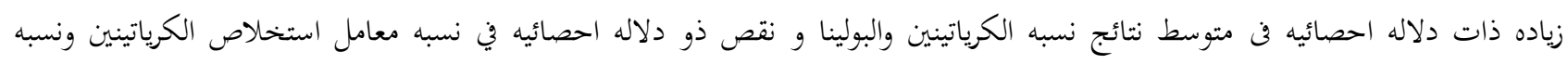

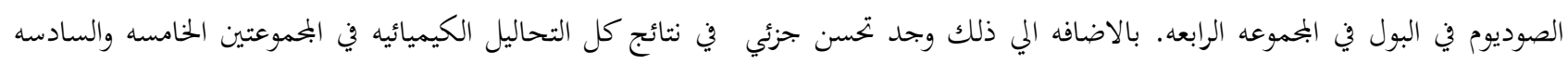

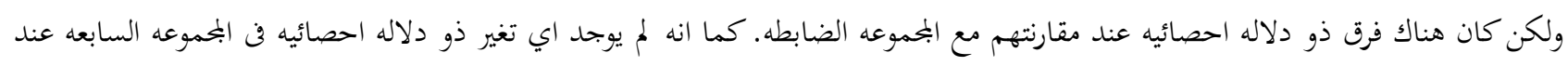

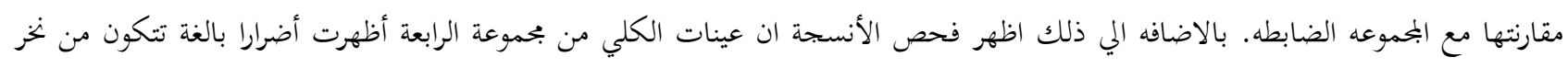

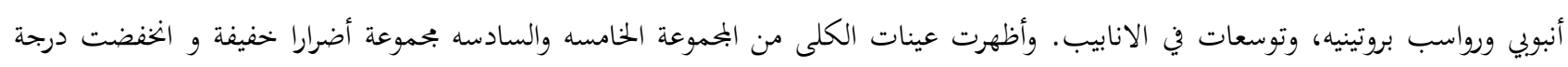
التغيرات المرضية بشكل ملحوظ بالمقارنة مع المحموعة الرابعة. وأظهرت مقاطع من المجموعة السابعة تحسنا كبيرا ملحوظا في كافة التغييرات.

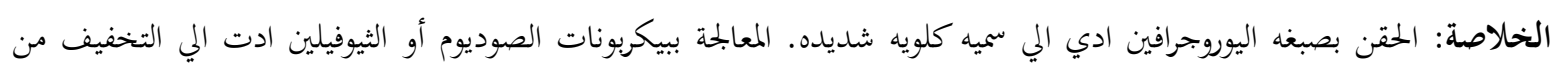

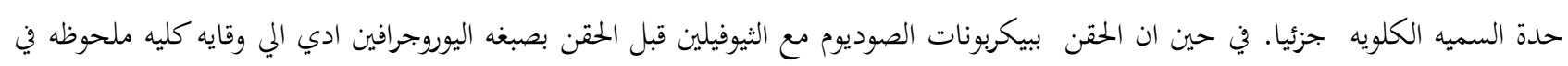
وظيفة الكلى والفحص الهيستوباثولوجي في الفئران.

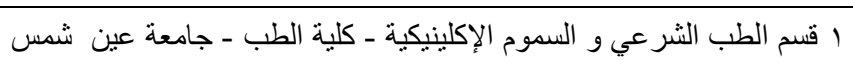

